\title{
Diluent Dispersion in Poly(vinyl chloride)-Plasticizer Systems
}

\author{
Noriyuki Kinjo and Tsurutaro NakagaWA \\ Department of Polymer Science, Faculty of Science, \\ Hokkaido University, Sapporo, Japan.
}

(Received June 25, 1973)

\begin{abstract}
Dynamic mechanical and dielectric measurements were carried out on plasticized poly(vinyl chloride) (PVC). The complex shear moduli $\left(G^{\prime}\right.$ and $\left.G^{\prime \prime}\right)$ in the temperature range $-196-+100^{\circ} \mathrm{C}$ were measured by means of a free-oscillating torsional pendulum at about $0.3 \mathrm{~Hz}$. The viscoelastic behavior of plasticizers was studied by the TBA method. The added plasticizers were tricresyl phosphate (TCP), butyl benzyl phthalate (BBP), dioctyl phthalate (DOP), dibutyl sebacate (DBS), and dioctyl sebacate (DOS).

The effects of plasticizers on the $\alpha-\beta$-, and $\gamma$-dispersions of PVC appear in different ways. The $\alpha$-dispersion due to the glass transition shifts to a lower temperature as the plasticizer content increases. The $\beta$-dispersion is completely suppressed by the presence of a small amount $(16 \mathrm{wt} \%)$ of plasticizers. A new dispersion labeled as $\gamma$, which is not observed for both unplasticized PVC and pure plasticizers, appears in these mixed systems with the exception of the PVC-TCP system. This is an example of the socalled "diluent dispersion". The intensity of the $\gamma$-dispersion increases with increasing plasticizer content and shows a saturation tendency. The appearance of this dispersion is highly sensitive to the nature of the additives and the ability of plasticizers to provoke this dispersion is in the order of DBS $>$ DOS $>$ DOP $>$ BBP. The $\gamma$-dispersion is also observed in the dielectric results and the activation energy is estimated to be $6.5 \pm 0.5 \mathrm{kcal} / \mathrm{mol}$.

\section{KEY WORDS Diluent Dispersion / Plasticizer / Poly(vinyl chloride) /} Viscoelasticity /
\end{abstract}

Although numerous investigations have been made concerning the low-temperature relaxation behavior of polymers, ${ }^{1-2}$ the majority of these studies are devoted to pure polymers such as amorphous and crystalline ones. Relatively few reports direct their interest to the effect of added diluents. The well-known example is the socalled "water dispersion" of some nylons swollen with water. $^{1-5}$ Recently Janáček, et al., ${ }^{6-8}$ paid special attention to the swelling effect of various diluents on the poly(methacrylic acid) esters.

The relaxation behavior of polymer-diluent systems is quite complex and they have been generally interpreted in terms of the polymerdiluent interactions. Some dispersions of pure polymers are suppressed ${ }^{9-11}$ and/or shifted to a higher temperature by the presence of diluents. $^{6-8,12}$ In some cases, the creation of new peaks is observed. ${ }^{14,15}$ At any rate, there are many points which are not obvious in the viscoelastic behavior of the polymers swollen with some diluents.

The purpose of the present work is to show how the interaction between PVC and some low-molecular-weight substances influences the relaxation behavior of these mixed systems. Especially we are interested in the "diluent dispersion" of these systems.

\section{EXPERIMENTAL}

\section{Samples}

Commercial PVC, a product of Denki Kagaku Chemical Industries Co., Ltd., having an average degree of polymerization of 1100 , was used. This PVC powder was blended with various amounts of plasticizers and small amounts of stabilizers, calendered in a hot roll for $10 \mathrm{~min}$ at $170^{\circ} \mathrm{C}$, and compression-molded into sheets of $0.5 \mathrm{~mm}$ in thickness. The plasticizers used were all commercial materials: tricresyl phosphate (TCP), butyl benzyl phthalate (BBP), dioctyl(di- 
2-ethylhexyl) phthalate(DOP), dibutyl sebacate (DBS), and dioctyl(di-2-ethylhexyl) sebacate (DOS). The plasticizer contents were 16, 32, 40 , and $59 \mathrm{wt} \%$.

\section{Measurements}

The complex shear moduli $\left(G^{\prime}\right.$ and $\left.G^{\prime \prime}\right)$ in the temperature range $-196-100^{\circ} \mathrm{C}$ were measured for the strip-shaped specimens $\left(0.5 \times 12 \times 60 \mathrm{~mm}^{3}\right)$ by a free-oscillating torsional pendulum at about $0.3 \mathrm{~Hz}$.

The dielectric constant and loss were measured with a transformer bridge (Ando Denki TR-1B) at constant frequencies of $1,3,10$, and $30 \mathrm{kHz}$.

The viscoelastic behavior of the plasticizers was studied by means of TBA (torsional braid analysis) method. ${ }^{17}$ After dipping a glass braid into the liquid plasticizers, these plasticizer-braid systems were mounted on the torsional pendulum apparatus, quickly immersed in liquid nitrogen and then damping measurements were made. The complex elastances of these systems are obtained, but not the elasticities of plasticizers by this technique. Since the glass braid shows no mechanical dispersion, as confirmed previously, the observed relaxation phenomena are considered to be due to the characteristics of the impregnating compound itself.

\section{RESULTS AND DISCUSSION}

The results of viscoelastic measurements are shown in Figures $1-5$, where the dynamic shear moduli $\left(G^{\prime}\right.$ and $\left.G^{\prime \prime}\right)$ are plotted against temperature at various plasticizer contents.

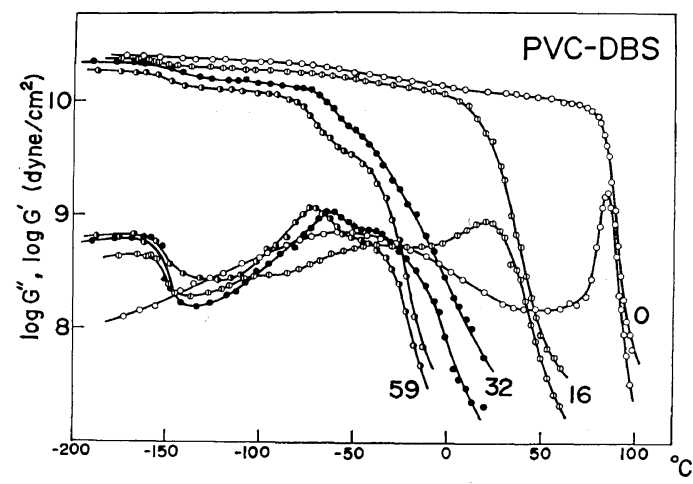

Figure 1. Temperature dependence of $G^{\prime}$ and $G^{\prime \prime}$ for the PVC plasticized with DBS. The numbers in this Figure are the DBS concentrations (wt\%).

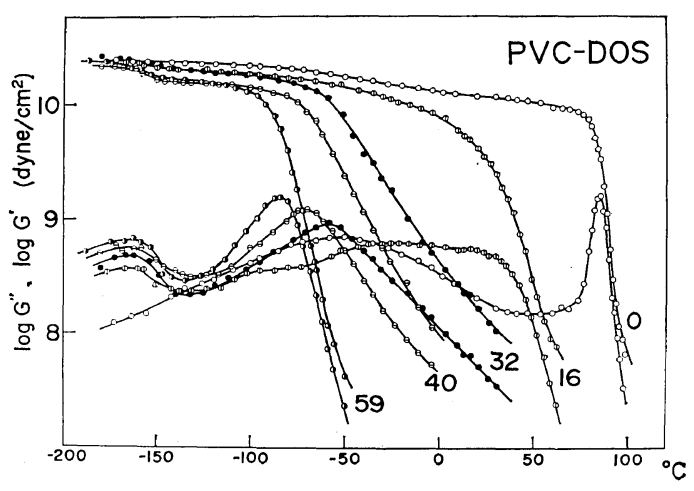

Figure 2. Temperature dependence of $G^{\prime}$ and $G^{\prime \prime}$ for the PVC plasticized with DOS. The numbers in this Figure are the DOS concentrations (wt \%).

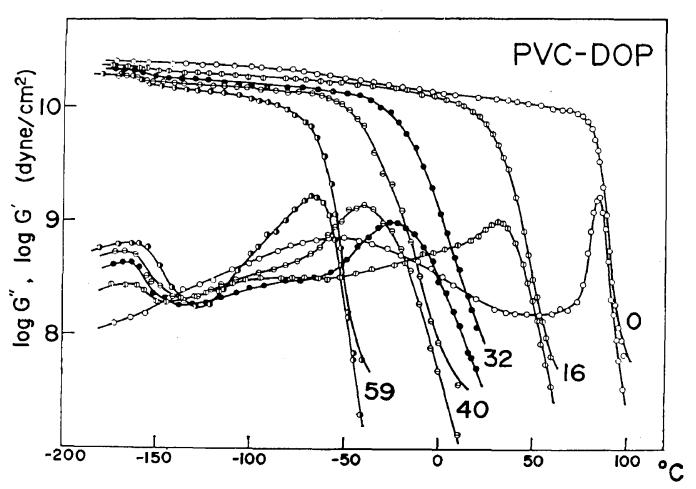

Figure 3. Temperature dependence of $G^{\prime}$ and $G^{\prime \prime}$ for the PVC plasticized with DOP. The numbers in this Figure are the DOP concentrations (wt \%).

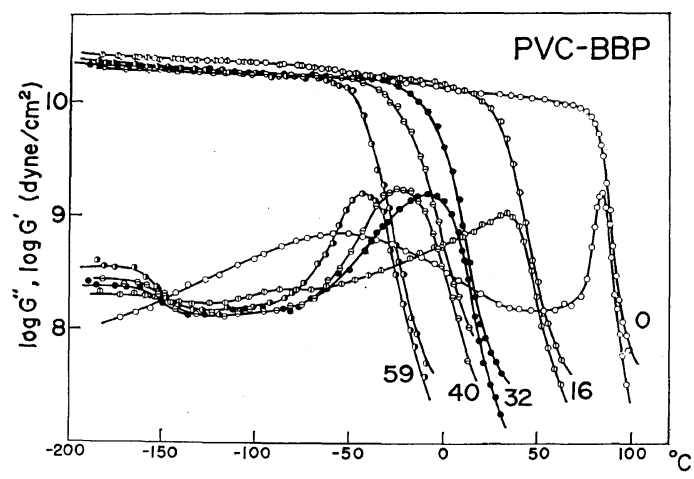

Figure 4. Temperature dependence of $G^{\prime}$ and $G^{\prime \prime}$ for the PVC plasticized with BBP. The numbers in this Figure are the BBP concentrations (wt\%). 


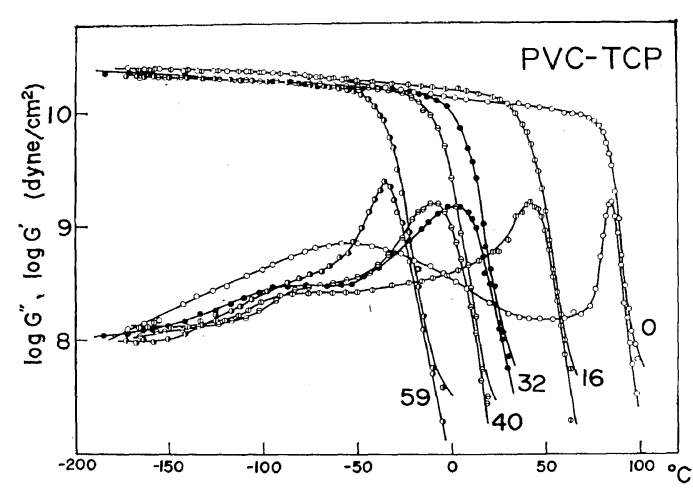

Figure 5. Temperature dependence of $G^{\prime}$ and $G^{\prime \prime}$ for the PVC plasticized with TCP. The numbers in this Figure are the TCP concentrations (wt\%).

Two dispersion regions are distinguishable in the case of unplasticized PVC and are labelled as $\alpha$ and $\beta$ in order of decreasing temperature. In the PVC-plasticizer systems, a new dispersion near $-165^{\circ} \mathrm{C}$ was observed and we designate it as $\gamma$. The effects of added plasticizers on these dispersions will be described in the following sections.

\section{The Effect on the $\alpha$-Dispersion}

The $\alpha$-dispersion is associated with the glass transition of these systems. The plasticizing effect of additives is seen as resulting in the shift of the $\alpha$-relaxation region to the lower temperature region. These features are illustrat-

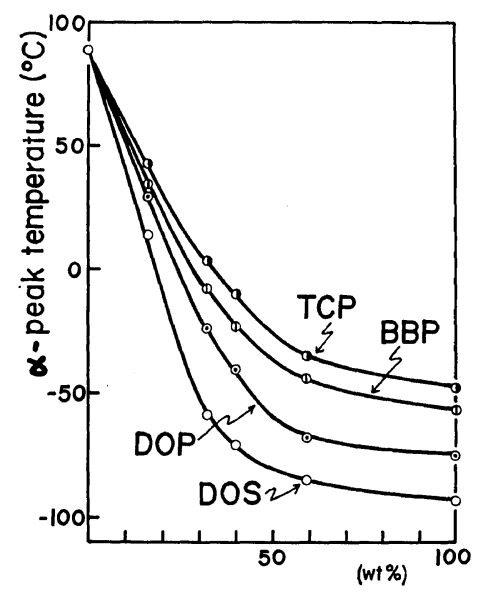

Figure 6. The $\alpha$-peak temperatures of the PVCplasticizer systems plotted against plasticizer content. ed in Figure 6, where the $\alpha$-peak temperatures are plotted against plasticizer content. The loss peak temperatures due to the glass transition of pure plasticizers were determined by the TBA method (cf. Figures 7-11). As seen from Figure 6, the $\alpha$-peak temperatures of the plasticized PVC fall rapidly as the plasticizer contents increase up to $50 \mathrm{wt} \%$ and then decrease gradually. The lower the glass transition temperature of plasticizer, the stronger is the plasticizing effect.

The mechanical behavior in this region is not simple; that is, the transition region broadens at first $^{13}$ and becomes narrower at higher plasticizer content as shown in Figures $1-5$. This finding agrees with the results of the mechanical, ${ }^{18,19}$ the dielectric, ${ }^{20}$ and the heat capacity measurements. ${ }^{21}$

As long as some diluents like plasticizers are homogeneously dispersed in the polymer matrix, the glass transition of the diluents themselves cannot be observed, Illers ${ }^{16}$ showed that internal friction peaks due to the glass transition of diluents appear in his polystyrene samples in whose matrix alcohols were embedded heterogeneously. Shen, et al., ${ }^{14}$ carried out viscoelastic measurements for the poly(methyl methacrylate) (PMMA)-dioctyl adipate (DOA), and reported that the loss peak due to the glass transition of DOA itself was observed at the higher concentrations of DOA, but not at the lower DOA concentrations. Quoting the Illers' results, they considered that clustering of DOA molecules is possible at the higher DOA concentrations.

In the work reported here, the loss maxima due to the glass transitions of the plasticizers themselves were not observed for the PVCplasticizer systems as illustrated in Figures $1-5$. From these facts we consider that the plasticizer molecules are homogeneously solvated in the PVC matrix up to a concentration of $50 \mathrm{wt} \%$. The domain formation of plasticizers might take place in these samples above this concentration. However, if so, one cannot distinguish the loss peak of the plasticizer from that of the plasticized PVC, since the $\alpha$-dispersion region of the plasticized PVC is situated in the glass transition region of diluents as seen in Figure 6.

In the case of PVC-DBS (Figure 1), a new 
dispersion was observed as a shoulder in the region of $-40^{\circ} \mathrm{C}$. The temperature location of this dispersion is independent of the DBS concentration. A possible mechanism for this dispersion is the melting of the DBS domain which may present in the PVC matrix as a suspended cluster, if one takes into consideration the fact that, as will be pointed out later, the DBS is very crystallizable substance. This idea is tentative and the details are not clear, for there is some difference between the temperature of this dispersion and the melting temperature (about $-11^{\circ} \mathrm{C}$ ) of DBS.

The Effect on the $\beta$-Dispersion

It is generally recognized that the $\beta$-dispersion of PVC is attributed to the local mode motion of the main chain. ${ }^{22}$ The loss maximum occurs at $-55^{\circ} \mathrm{C}(0.3 \mathrm{~Hz})$. The temperature dependence of the relaxation time conforms to the Arrhenius type relationship and the apparent activation energy has been estimated to be $14.3 \mathrm{kcal} / \mathrm{mol}^{9}$

The plasticizers exert a peculiar effect on this dispersion. With increasing plasticizer content, this peak decreases in magnitude and shifts to a lower temperature region, ${ }^{9}$ and then completely disappears at about $16 \mathrm{wt} \%$ of plasticizer content as, shown in Figures $1-5$. This behavior is observed significantly in the PVC-TCP, PVCBBP, and PVC-DOP systems. In the other two systems, however, the disappearance of the

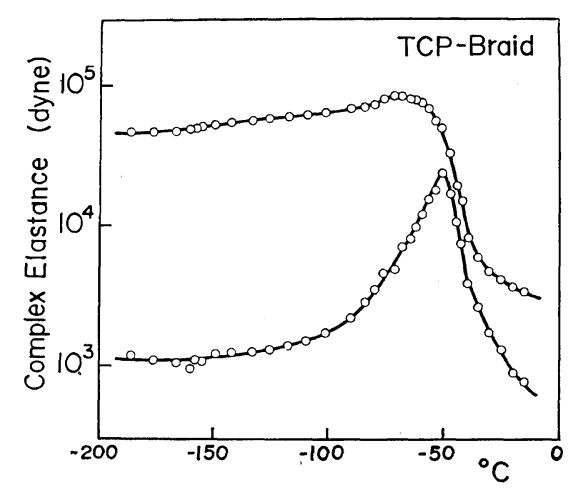

Figure 7. Temperature dependence of the complex elastance for TCP-Braid system. The upper and the lower curves correspond to the real part and to the imaginary part of the complex elastance respectively. $\beta$-dispersion is not obvious because of the onset of the $\alpha$-dispersion. The suppressing effect of plasticizers on the $\beta$-dispersion has been adequately discussed previously ${ }^{9}$ in connection with the "antiplasticization" in the slightly plasticized PVC.

\section{The Effect on the $\gamma$-Dispersion}

The $\gamma$-dispersion, which is absent in the case of unplasticized PVC, is observed in the PVCplasticizer systems. Whether the plasticizers show a loss maximum in this temperature region or not, it must be examined at first.

We carried out the viscoelastic measurements

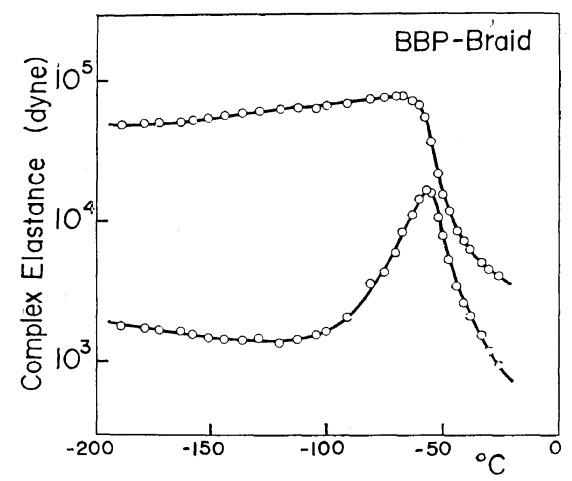

Figure 8. Temperature dependence of the complex elastance for BBP-Braid system. The upper and the lower curves correspond to the real part and to the imaginary part of the complex elastance respectively.

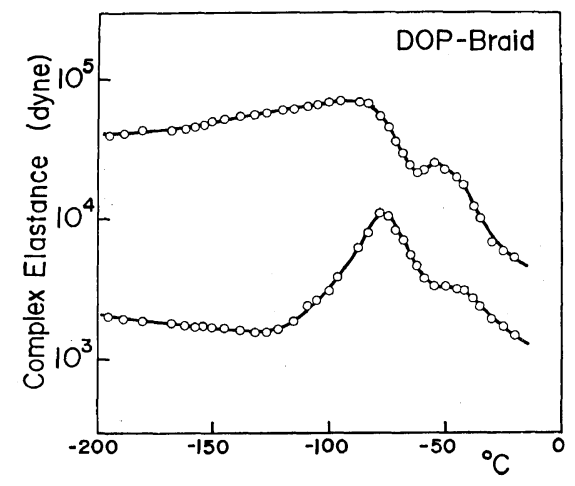

Figure 9. Temperature dependence of the complex elastance for DOP-Braid system. The upper and the lower curves correspond to the real part and to the imaginary part of the complex elastance respectively. 


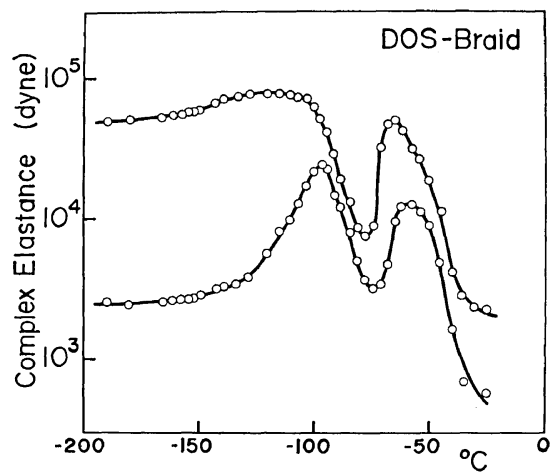

Figure 10. Temperature dependence of the complex elastance for DOS-Braid system. The upper and the lower curves correspond to the real part and to the imaginary part of the complex elastance respectively.

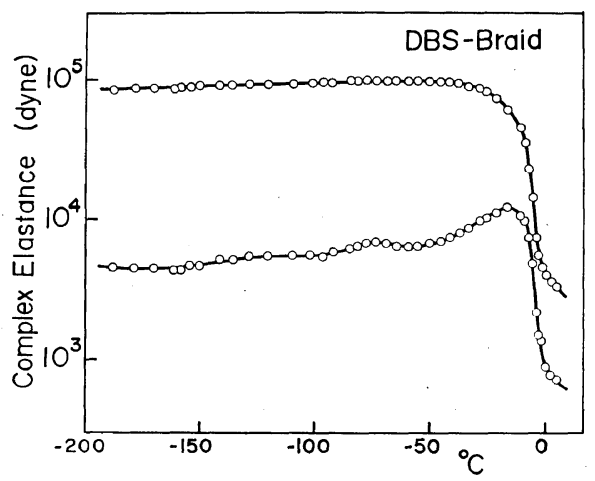

Figure 11. Temperature dependence of the complex elastance for DBS-Braid system. The upper and the lower curves correspond to the real part and to the imaginary part of the complex elastance respectively.

for the plasticizers by the TBA method. ${ }^{17}$ The samples were quickly cooled to a solid and then the temperature was gradually raised. Interesting results were obtained and these are shown in Figures 7-11. The TCP and the BBP show a single mechanical transition which is associated with the glass transition. These plasticizers are vitrifiable substances. In the case of DOS (Figure 10), a decrease in the storage modulus occurs at the glass transition between $-100-$ $-80^{\circ} \mathrm{C}$. A subsequent increase in modulus is observed, which indicates the crystallization of the sample in the glass braid. The final decrease near $-45^{\circ} \mathrm{C}$ is due to the melting of crystals.
Figure 9 (DOP) shows an intermediate feature between those of TCP and DOS. The DBS is a very crystallizable substance and shows no glass transition. A decrease in storage modulus due to the fusion is observed at about $-11^{\circ} \mathrm{C}$.

The most important finding in Figures 7-11 is that the plasticizers show no loss maximum in the temperature region from $-196^{\circ} \mathrm{C}$ to their glass transition temperatures, and therefore this new $\gamma$-dispersion cannot be attributed to the diluent molecules alone. Consequently it is of interest that the peculier $\gamma$-dispersion appears in the mixed systems of PVC and plasticizers, whereas both components are viscoelastically inactive in this temperature region.

Shen, et al. ${ }^{15}$ emphasized that the mechanical properties of plasticized polymers in the glassy state cannot be predicted on the basis of either the polymers or the diluents alone. The term "diluent dispersion", in spite of having no clear

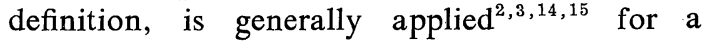
certain dispersion which appears in polymers swollen with some low-molecular-weight substances. The $\gamma$-dispersion of our samples must be an example of the "diluent dispersion".

The $\gamma$-peak is located near $-165^{\circ} \mathrm{C}$ at about $0.3 \mathrm{~Hz}$, though the accurate positions cannot be determined because of their flatness. The position of the $\gamma$-dispersion is independent of the plasticizer contents and its intensity becomes stronger with the increase in plasticizer content with the exception of PVC-TCP systems. The loss moduli of the $\gamma$-peaks, $G_{r}{ }^{\prime \prime}$, are plotted as a function of plasticizer contents in Figure 12.

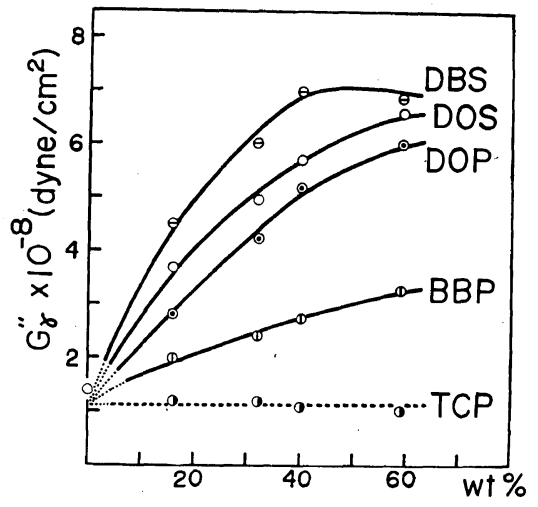

Figure 12. The loss modulus of the $\gamma$-peak of plasłicized PVC plotted against plasticizer content. 
The dependence of the $G_{r}^{\prime \prime}$ values on the plasticizer contents is not linear and shows a saturation tendency. In the case of PVC-TCP, the values of the loss moduli in this temperature region are lower than that of the unplasticized PVC owing to two reasons; the $\beta$-dispersion of PVC disappears in the presence of plasticizers and the PVC-TCP systems do not show the $\gamma$-dispersion.

These facts mentioned above suggest that the $\gamma$-dispersion originates from the interaction between PVC and the diluents. Moreover the appearance of this dispersion seems to be highly sensitive to the nature of the additives.

The ability of plasticizer to provoke the $\gamma$ dispersion is in the order of

$$
\text { DBS }>\text { DOS }>\text { DOP }>\text { BBP , TCP . }
$$

Although the TCP is incapable of bringing about the $\gamma$-dispersion, we put it in this sequence for the convenience of later discussion.

We intend to discuss the $\gamma$-dispersion from the viewpoint of the chemical structure of added plasticizers. Note that the inactive TCP is mainly composed of three benzene rings, but not of alkyl chains. The BBP which has a short butyl group and two benzene rings is weak in the effect of giving rise to the $\gamma$ dispersion. The effective DOP consists of two octyl groups and a benzene ring. The other plasticizers (DBS and DOS) which have long alkyl chains and no benzene ring are more effective in bringing about the $\gamma$-dispersion. Consequently the above order shows that the intensity of the $\gamma$-peak increases as the alkyl chain length of the diluents increases. On the basis of the above discussion, the $\gamma$-dispersion in the PVC-plasticizer systems could conceivably be attributed to the molecular motion of the alkyl chain sequences in the plasticizers under the condition that the plasticizers do exist in the PVC matrix.

It is contrary to the above expectation that the DBS is more effective than the DOS, because the alkyl chain length of the latter is longer than that of the former. This fact may be due to the branching of the DOS; the branching parts of the DOS hinder the molecular motion of whole alkyl chains in DOS more markedly compared to the case of DBS.

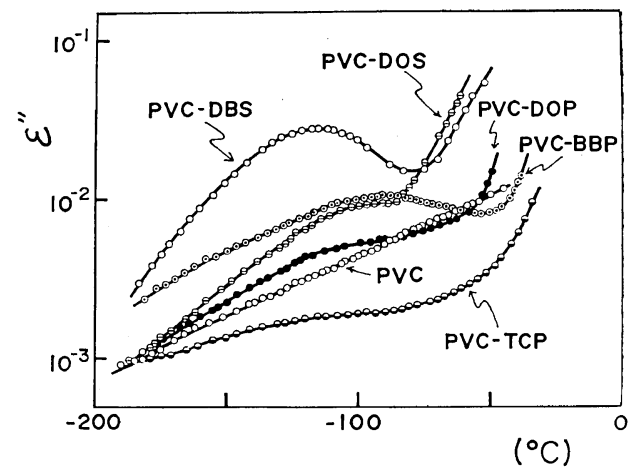

Figure 13. Temperature dependence of dielectric loss for the plasticized (40 wt \%) PVC at $10 \mathrm{kHz}$.

The dielectric results for the plasticized (40wt\%) PVC are shown in Figure 13. The $\varepsilon^{\prime \prime}$ of the unplasticized PVC gradually increases with increasing temperature, which corresponds to the lower temperature side of the $\beta$-dispersion. The $\varepsilon^{\prime \prime}$ values in the PVC-TCP system are lower than those of unplasticized PVC because of the disappearance of the $\beta$-dispersion and of the absence of the $\gamma$-dispersion. For the other systems the $\gamma$-dispersion appears as a peak or as a shoulder. These results coincide with our mechanical ones. But the effectiveness of plasticizers for the $\gamma$-peak intensity in the dielectric observations is not similar to that in mechanical ones. This discrepancy is not ex-

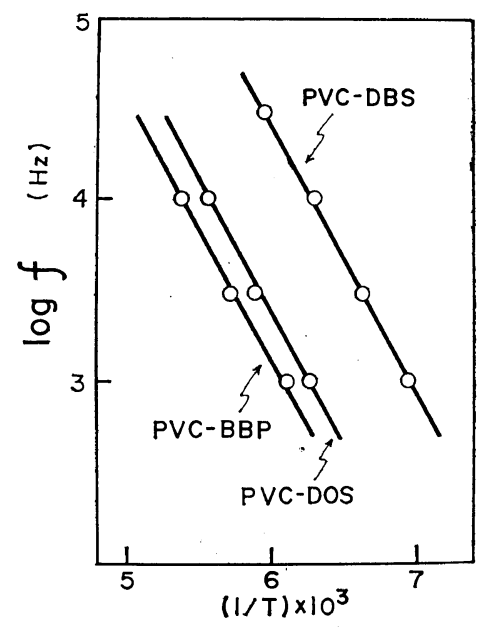

Figure 14. Logarithmic frequency of dispersion plotted against reciprocal absolute temperature of dielectric loss maximum ( $\gamma$-dispersion). 
plained clearly at present and is left unsolved for future study. At any rate, this finding in the dielectric experiments appears to prove that the polar groups, in addition to the nonpolar alkyl chain sequences, are essentially responsible for this $\gamma$-dispersion.

The transition maps are shown in Figure 14. The activation energy was estimated to be $6.5 \pm 0.5 \mathrm{kcal} / \mathrm{mol}$, which is independent of the systems studied. This value closely resembles the activation energy found for the relaxation process of the side-chain alkyl groups in the poly(methacrylic acid) esters. ${ }^{23,24}$

Janáček, et al., assumed that the low-temperature dispersions of pure polymers are transformed into another dispersions by the presence of diluents; for example, ${ }^{6,7}$ the transformation from the $\gamma$-dispersion of dried nylons to the $\beta$-dispersion of wet nylons, and from the $\gamma$-dispersion of pure poly(hydroxyethyl methacrylate) (PHEMA) to the $\beta$-dispersion of PHEMA containing low molecular organic substances.

Judging from the concentration dependence of both $\gamma$ and $\beta$-dispersion of our samples, it is impossible to suppose transformation from the $\beta$-dispersion to the $\gamma$-dispersion due to the presence of plasticizers.

There is another possibility for the transformation. Goldstein, et al., ${ }^{25}$ reported that secondary dispersions are observed for mixtures of low-molecular-weight substances in the glassy state. The secondary dispersions of low-molecular-weight substances in the glassy state were also observed for some alcohols by Illers ${ }^{16}$ and for enantholactone by Koleske, et al. ${ }^{26}$ These results suggest that the plasticizers used in this study may have some dispersions at cryogenic temperatures. However, it is not confirmed at present and is the subject for future study. Therefore, we cannot exclusively conclude that the $\gamma$-dispersion of the PVC-plasticizer systems results from the transformation of the secondary dispersion of plasticizers themselves.

Shen, et al., ${ }^{15}$ proposed a "dual mechanism" for the interpretation of "diluent peak". This idea is not applicable to our cases.

\section{CONCLUSION}

The most important finding reported in this paper is that a new "diluent dispersion" $(\gamma-$ dispersion) is observed for the PVC plasticized with some diluents in the low-temperature region where neither of the components shows a loss maximum. It is some interaction between diluents and PVC matrix that bring about the $\gamma$-dispersion. The appearance of this dispersion depends upon the diluent concentration as well as on the species of the diluents. Low-temperature $\gamma$-relaxation seems to be assigned to the cooperative motion of the alkyl chains in the diluents including some motion of the polar groups.

Our observation is concerned with one example of the diluent dispersion, and we believe that the accumulation of more data in regard to the "diluent dispersion" is necessary at present and it will lead to an adequate interpretation for its mechanism.

Acknowledgements. The authors wish to thank Dr. M. Kikkawa and Mr. S. Kawada of the Hitachi Electric Co., Ltd., for the supplying samples, and also wish to express their gratitude to Associate Professor T. Komatsu and Mr. K. Nakamura of Hokkaido University for their encouragement and discussions during the course of the present study. The authors wish to thank Professor T. Hideshima and Miss M. Kakizaki of Hokkaido University for their interest in our work and for affording facilities for the dielectric measurements. The present work has been supported in part by a Grant for Scientific Research from the Ministry of Education.

\section{REFERENCES}

1. A. E. Woodward and J.A. Sauer, "Physics and Chemistry of the Organic Solid State," Vol. II, D. Fox, M. M. Labes, and A. Weissleger, Ed., Interscience Publishers, New York, N. Y., 1965, p 637.

2. N. G. McCrum, B. F. Read, and G. Williams, "Anelastic and Dielectric Effects in Polymeric Solids," John Wiley \& Sons, Inc., New York, N. Y., 1967, p 478.

3. J. Kolařik and J. Janáček, J. Polym. Sci., Part C, 16, 441 (1967).

4. D. C. Prevorsek, R. H. Butler, and H.K. Reimschuessel, ibid., Part A-2, 9, 867 (1971).

5. Y.S. Papir, S. Kapur, C.E. Rogers, and E. 
Baer, ibid., Part A-2, 10, 1305 (1972).

6. J. Janáček, ibid., Part C, 23, 373 (1968).

7. J. Janáček and J. D. Ferry, J. Macromol. Sci. Phys., B5, 219 (1971).

8. F. Lednický and J. Janáček, ibid., B5, 335 (1971).

9. N. Kinjo and T. Nakagawa, Polymer J., 4, 143 (1973).

10. L. M. Robeson, Polym. Eng. Sci., 9, 277 (1969).

11. M. G. Wyzgoski and G. Soh-Yu Yen, Polymer J., 4, 29 (1973).

12. H. Jacobs and E. Jenckel, Makromol. Chem., 47, 72 (1961).

13. N. Kinjo, T. Komatsu, and T. Nakagawa, Nippon Kagaku Zasshi (J. Chem. Soc. Japan), 92, 27 (1971).

14. M. C. Shen and E.H. Cirlin, J. Macromol. Sci. Phys., B4, 293 (1970).

15. M. C. Shen and J. D. Strong, J. Appl. Phys., 38, 4197 (1967).

16. K. H. Illers, Rheol. Acta, 3, 186, 194 (1964).
17. A. F. Lewis and J. K. Gillham, J. Appl. Polym. Sci., 7, 685 (1963).

18. F. Würstlin, Kolloid Z., 113, 18 (1949).

19. K. Wolf, Kunststoffe, 41, 89 (1951).

20. E. R. Fitzgerald and R. F. Miller, J. Colloid Sci., 8, 148 (1953).

21. L. Ya. Martynenko, I. B. Rabinovich, Yu. V. Ovchinnikov, and V. A. Maslova, Vysokomol. Soedin., Ser. A, 12, 841 (1970).

22. N. Saito, K. Okano, S. Iwayanagi, and T. Hideshima, "Solid State Physics," Vol. 14, F. Seitz and D. Turnbull, Ed., Academic Press, New York, N. Y., 1963, p 387.

23. H. Ochiai, H. Shindo, and H. Yamamura, $J$. Polym. Sci., Part A-2, 9, 431 (1971).

24. Y. Kawamura, S. Nagai, J. Hirose, and Y. Wada, ibid. Part A-2, 7, 1559 (1969).

25. G. P. Johari and M. Goldstein, J. Chem. Phys., 53, 2372 (1970).

26. J. V. Koleske and R. D. Lundberg, J. Polym. Sci., Part A-2, 10, 323 (1972). 